\title{
Long-term effect of ropivacaine nanoparticles for sciatic nerve block on postoperative pain in rats
}

\author{
This article was published in the following Dove Press journal: \\ International Journal of Nanomedicine \\ 17 May 2016 \\ Number of times this article has been viewed
}

\author{
Zi Wangl,* \\ Haizhen Huang ${ }^{2, *}$ \\ Shaozhong Yang' \\ Shanshan Huang' \\ Jingxuan Guo' \\ Qi Tang' \\ Feng $\mathrm{Q}^{\mathrm{i}}$
}

'Department of Anesthesiology, Qilu Hospital of Shandong University, ${ }^{2}$ Department of Anesthesiology, Stomatology Hospital of Shandong University, Jinan, Shandong, People's Republic of China

*These authors contributed equally to this work
Correspondence: Feng Qi

Department of Anesthesiology, Qilu

Hospital of Shandong University,

107 Wenhua Xi Road, Jinan 2500I2,

Shandong, People's Republic of China

Tel +86 I85 60083756

Fax +86 53I 88692544

Email qifeng6632I@sina.com
Purpose: The analgesic effect of ropivacaine (Rop) for nerve block lasts only $\sim 3-6$ hours for single use. The aim of this study was to develop long-acting regional anesthetic Rop nanoparticles and investigate the effects of sciatic nerve block on postoperative pain in rats.

Materials and methods: Rop nanoparticles were developed using polyethylene glycol-co-polylactic acid (PELA). One hundred and twenty adult male Wistar rats were randomly divided into four groups ( $\mathrm{n}=30$, each): Con (control group; $0.9 \%$ saline, $200 \mu \mathrm{L}$ ), PELA (PELA group; $10 \mathrm{mg}$ ), Rop (Rop group; 0.5\%, $200 \mu \mathrm{L}$ ), and Rop-PELA (Rop-PELA group; $10 \%, 10 \mathrm{mg}$ ). Another 12 rats were used for the detection of Rop concentration in plasma. The mechanical withdrawal threshold and thermal withdrawal latency were measured at 2 hours, 4 hours, 8 hours, 1 day, 2 days, 3 days, 5 days, and 7 days after incision. The expression of c-FOS was determined by immunohistochemistry at 2 hours, 8 hours, 48 hours, and 7 days. Nerve and organ toxicities were also evaluated at 7 days.

Results: The duration of Rop absorption in the plasma of the Rop-PELA group was longer ( $>8$ hours) than that of the Rop group ( 4 hours). Mechanical withdrawal threshold and thermal withdrawal latency in the Rop-PELA group were higher than that in other groups ( 4 hours-3 days). c-FOS expression in the Rop-PELA group was lower than that in the control group at 2 hours, 8 hours, and 48 hours and lower than that in the Rop group at 8 hours and 48 hours after paw incision. Slight foreign body reactions were observed surrounding the sciatic nerve at 7 days. No obvious pathophysiological change was found in the major organs after Rop-PELA administration at 7 days.

Conclusion: Rop-PELA provides an effective analgesia for nerve block over 3 days after single administration, and the analgesic mechanism might be mediated by the regulation of spinal c-FOS expression. However, its potential long-term tissue toxicity needs to be further investigated.

Keywords: polyethylene glycol-co-polylactic acid, nanoparticle, ropivacaine, sciatic nerve block, postoperative pain

\section{Introduction}

Acute postoperative pain continues to be a major problem despite the safety of the drugs used in the perioperative period. ${ }^{1}$ Effective control of acute postsurgical pain is essential for the patient. It can not only contribute to several clinically valuable outcomes, including earlier patient mobilization and quicker recovery, which can result in a shortened hospital stay and reduced costs, but also prevent the development of chronic pain in the long term, which can occur if early acute pain is prolonged. $^{2-6}$ Besides providing excellent analgesia at rest and in movement, the opioid-sparing effects of local anesthetics are associated with reduced opioid-related side effects. $^{7}$ 
Polyethylene glycol-co-polylactic acid (PELA) is an amphipathic, nonionic polymer with certain amounts of hydrophilic polyethylene glycol (PEG) segments. It has been investigated as a delivery system for drugs, protein, and antigen. Govender et al compared the influence of formulation variables on particle size, physicochemical properties, and incorporation efficiency of PELA nanoparticle and polylacticco-glycolic acid (PLGA) nanoparticle loaded with procaine; the results indicated that PELA nanoparticle could prolong circulation time and decrease liver uptake. ${ }^{8}$ PEG-PLGA nanoparticles could extend half-life of bovine serum albumin loaded in PLGA from 13.6 minutes to 4.5 hours. ${ }^{9}$ Studies have also shown that the copolymer of PELA was a good drug carrier. ${ }^{10-12}$ It was beneficial to improve the affinity of the matrix polymer and drugs, which might result in an increase in entrapment efficiency and a decrease in the burst release effect to achieve a stable and sustained-release profile. ${ }^{11}$

There is no ideal agent available to better manage the acute pain during the first few days (3-5 days) after surgery. ${ }^{13}$ Currently, peripheral nerve block with ropivacaine (Rop), one of the safest local anesthetics in the market, is widely used in the management of acute pain..$^{14,15}$ The analgesic effect of Rop lasts $\sim 3-6$ hours after intrathecal injection for single use. However, the analgesic benefits of even the longest acting local anesthetics at present are limited to $<6$ hours when placed subcutaneously and to $<20$ hours for a typical femoral nerve block. ${ }^{16}$ Development of a long-acting and safe local anesthetic for clinical single use is necessary. Previous data shows that single injection of bupivacaine in poly(D,L:lactic acid-co-castor oil) 3:7 caused sensory block in mice that lasted 48 hours. ${ }^{17,18}$ In our previous study, the PELA copolymers were well developed and their released characterization was $\sim 1$ week in vitro. ${ }^{19}$ However, little information is available about the Rop-loaded nanoparticle (Rop-PELA) and its effects for sciatic nerve block on postoperative pain in vivo. The current study was designed to developed longacting Rop-PELA nanoparticles and observe the analgesic effects for nerve block in a rat postoperative pain model.

\section{Materials and methods Preparation of nanoparticles}

Polymers of PELA (PEG:polylactic acid =1:35) were synthesized by ring-opening polymerization of D, Llactide in the presence of PEG. Rop-PELA nanoparticles $(10 \%, w / w)$ were synthesized by o/w emulsion solvent evaporation method and measured in the laboratory of School of Chemistry and Chemical Engineering of Shandong
University. The copolymers were determined using the methods of Fourier transform infrared spectroscopy, nuclear magnetic resonance, thermogravimetric analysis, and gel permeation chromatography. ${ }^{20}$ The average molecular weight of Rop-PELA was $83 \mathrm{kDa}$ (PEG: $2 \mathrm{kDa}$ ), and the average particle size of Rop-PELA was $50 \mu \mathrm{m}$. Rop release of Rop-PELA in vitro was measured using ultraviolet spectrophotometer.

\section{Animals}

The investigation was approved by the Animal Care and Use Committee of Shandong University. All animal experiments were performed in compliance with the Guiding Principles for the Care and Use of Laboratory Animals, Shangdong University, People's Republic of China. All efforts were made to minimize suffering and the number of animals used. Male Wistar rats weighing 250-300 g were housed three per cage with controlled relative humidity $(20 \%-30 \%)$ in a 12 -hour light-dark cycle at room temperature $\left(24^{\circ} \mathrm{C}\right)$. Food and water were freely accessed. Incision, behavioral testing, tissue isolation, and processing were carried out by the same person to reduce interindividual total variability. A total of 134 rats were used for this study (two were excluded from the study because of death after anesthesia). In order to minimize any changes in gene expression that might be affected by responses to noxious stimuli, two separate groups of rats $(\mathrm{n}=6$, each) were used for the determination of Rop in plasma. In all, 24 rats were used for behavior testing and the rest of them were sacrificed for immunohistochemistry. They were randomly divided into four groups (six rats at every time point). Data from all rats were included in the analysis.

\section{Drug application}

During chloral hydrate anesthesia $(10 \%, 3 \mathrm{~mL} / \mathrm{kg})$, a standardized surgical technique was used to expose the left sciatic nerve through a $1 \mathrm{~cm}$ skin incision in a manner similar to that reported by Brummett et al. ${ }^{21}$ The sciatic nerve was freed from its investing fascia, and saline $(0.9 \%, 200 \mu \mathrm{L})$, PELA (without Rop, 10 mg PELA), Rop $(0.5 \%, 200 \mu \mathrm{L}$, $1 \mathrm{mg}$ Rop diluted by saline), and Rop-PELA (10\%, $10 \mathrm{mg}$ nanoparticles, including $1 \mathrm{mg}$ Rop) were applied randomly peripherally to the surroundings of the sciatic nerve. PELA $(10 \mathrm{mg})$ and Rop-PELA $(10 \mathrm{mg})$ were in a white crystal state without dilution by saline and were delivered to the surroundings of the sciatic nerve with a small spoon. The surgical wounds were then closed in a standard manner with nylon suture. 


\section{Postoperative pain model}

A postoperative pain model was produced by a hind paw incision as described by Brennan et al. ${ }^{22}$ This procedure was performed immediately after the drug application. Briefly, rats were anesthetized with chloral hydrate $(10 \%, 3 \mathrm{~mL} / \mathrm{kg})$ via intraperitoneal injection. The plantar aspect of the left hind paw was prepared with povidone iodine, and a $1.0 \mathrm{~cm}$ longitudinal incision was made at a distance of $0.5 \mathrm{~cm}$ from the end of the heel. The plantar muscles were elevated using forceps and incised longitudinally from the muscle origin and insertion to remain intact. After hemostasis with gentle pressure, the wound was closed with two mattress sutures using 5-0 silk. The wounds were checked for evidence of dehiscence before behavioral testing. The hind paw incision immediately induced mechanical hypersensitivity in rats, as indicated by the reduced mechanical withdrawal threshold (MWT) in all groups. The rats were not screened in our study if they showed no response to MWT or thermal withdrawal latency (TWL). The person performing the behavioral testing was blinded to the drug administration.

\section{Pharmacokinetics analysis}

After chloral hydrate anesthesia, the rats were administered with Rop or Rop-PELA ( $\mathrm{n}=6$, each) for sciatic nerve blocking. In all, $0.7 \mathrm{~mL}$ of blood was sampled at 0.25 hours, 0.5 hours, 1 hour, 2 hours, 4 hours, 6 hours, 8 hours, 1 day, 2 days, 3 days, 5 days, and 7 days after administration. After each puncture, $0.5 \mathrm{~mL}$ of saline was injected by intraperitoneal routine to compensate blood loss. The samples were transferred to test tubes containing heparin and then centrifuged at 3,000 rpm for 10 minutes. The plasma samples were kept at $-20^{\circ} \mathrm{C}$. The plasma concentration of Rop was measured by high-performance liquid chromatography analysis, which was performed on a LC-10A system (Shimadzu, Kyoto, Japan), equipped with LC-10ATVP pumps, a Shimadzu SPD-10 AUV detector, and a Phenomenex Gemini C 18 column $(5 \mu \mathrm{m}$, $4.6 \times 250 \mathrm{~mm}$ ). The detection wavelength was set at $215 \mathrm{~nm}$, and the mobile phase solvent consisting of methanol and $0.01 \mathrm{M}$ phosphate-buffered saline (PBS) adjusted to $\mathrm{pH} 3.8$ $(35: 65, \mathrm{v} / \mathrm{v})$ was pumped at a flow rate of $1.2 \mathrm{~mL} / \mathrm{min}$. Bupivacaine hydrochloride was used as an internal standard. ${ }^{23}$

The pharmacokinetic parameters Rop and Rop-PELA in plasma, peak concentration $\left(C_{\max }\right)$, time to peak concentration $\left(T_{\text {max }}\right)$, absorption rate constant $\left(K_{\mathrm{a}}\right)$, absorption half-life $\left(T_{1 / 2}\right.$, abs), elimination half-life ( $T_{1 / 2}$, elimination), elimination rate constant $\left(K_{\mathrm{e}}\right)$, and area under the concentration-time curve (AUC) of the drug from the time of dosing to the end of the drug release were calculated.

\section{Behavioral testing}

Because the block did not comprise the motor nerves to the hip muscles, all animals were able to withdraw the tested paw in response to pain.

\section{Measurement of mechanical allodynia}

Mechanical allodynia was assessed using an electrical von Frey filament (BME-404; Biomedical Engineering Institute of the Chinese Academy of Medical Sciences, Beijing, People's Republic of China). ${ }^{24}$ Rats were acclimated to the testing environment and stimulated for 2 days. On the day of the experiment, rats were placed individually on an elevated wire mesh floor (grid $10 \times 10 \mathrm{~mm}$ ) covered with a clear plastic cage $(20 \times 25 \times 15 \mathrm{~cm})$ and allowed to adjust to the environment for 30 minutes. The steel filament $(d=0.4 \mathrm{~mm})$ was fixed to the handheld pressure transducer, and the tip was applied perpendicularly to the area adjacent to the wound with a gradual increase in pressure. The force needed to elicit the withdrawal of the hind paw was automatically recorded as MWT in grams by the pressure transducer. Stimulations were performed five times for each rat at 1 minute intervals, and the mean value of the five readings was used for the analysis as the individual threshold. Stimulations were performed before (pre) and after the hind paw incision ( 2 hours, 4 hours, 8 hours, 1 day, 2 days, 3 days, 5 days, and 7 days after application, $\mathrm{n}=6$ for each).

\section{Measurement of thermal hyperalgesia}

Thermal hyperalgesia was measured with Full-Automatic Plantar Analgesia Tester (BEM-410C; Biomedical Engineering Institute of the Chinese Academy of Medical Sciences) as described previously. ${ }^{24}$ Rats were placed individually on a glass floor $(3 \mathrm{~mm})$ covered with clear plastic cage and allowed to acclimate. TWL was assessed according to the method described by Hargreaves et al. ${ }^{25}$ In brief, the radiant heat underneath a glass floor was directed to the middle of incision. The end points in the test were the characteristic lifting or licking of the hind paw. The time to the end point was considered as TWL, which was shown digitally on a display screen. Before the experiments, the intensity of the heat was adjusted to produce TWL of $12-15$ seconds in the normal rats. An automatic 25 second cutoff was used to prevent tissue damage. Each rat was tested at least three times, at an interval of 5 minutes. The average of three trials was used to obtain TWL. Stimulations were performed before (pre) and after the hind paw incision ( 2 hours, 4 hours, 8 hours, 1 day, 2 days, 3 days, 5 days, and 7 days after application, 
$\mathrm{n}=6$ for each). All behavioral tests were conducted under blind conditions.

\section{Immunohistochemistry}

Rats were anesthetized with chloral hydrate $(10 \%, 3 \mathrm{~mL} / \mathrm{kg}$, intraperitoneal injection) and were subjected to sternotomy followed by intracardial perfusion with $0.01 \mathrm{M} \mathrm{PBS}$ (pH 7.4) and $400 \mathrm{~mL}$ of $4 \%$ paraformaldehyde in $0.1 \mathrm{M} \mathrm{PBS}$ at $4{ }^{\circ} \mathrm{C}$. The spinal cord was dissected out and post fixed in the same fixative for 48 hours at $4^{\circ} \mathrm{C}$. Serial dehydrations with graded ethanol and xylene were followed by embedding in paraffin. The spinal cord segments L4-L5 were cut transversely into $4 \mu \mathrm{m}$ sections. ${ }^{26}$

Immunohistochemistry was performed utilizing the labeled streptavidin-biotin method using a Three-Step IHC Detection Reagent (ZSGB-BIO, Beijing, People's Republic of China). The paraffin-embedded sections were heated for 2 hours at $68^{\circ} \mathrm{C}$, deparaffinized in xylene, and rehydrated through graded ethanol at room temperature. Microwave accentuation was used for 10 minutes with ethylenediaminetetraacetic acid buffer (1:50, pH =8.0, ZLI-9066; ZSGB-BIO). Then after two rinses in PBS, sections were put in a humid chamber and incubated in $0.3 \%$ hydrogen peroxide for 15 minutes. After three rinses in PBS, the sections were treated for 30 minutes at $37^{\circ} \mathrm{C}$ with normal goat serum (I9001; ZSGB-BIO) and incubated overnight at $4^{\circ} \mathrm{C}$ with c-FOS antibody (diluted 1:300 in PBS; ImmunoWay, Newark, DE, USA). Again after washing three times in PBS, the sections were incubated with biotinylated goat anti-rabbit serum (ZSGB-BIO) for 30 minutes at $37^{\circ} \mathrm{C}$. Then after three rinses, the sections were treated with horseradish peroxidase-labeled streptavidin complex (ZSGB-BIO) for 30 minutes at $37^{\circ} \mathrm{C}$. The reaction product was visualized with diaminobenzidine tetrahydrochloride substrate kit (ZSGB-BIO). Finally, after weakly counterstaining with hematoxylin, the sections were dehydrated and covered. As for negative controls, the sections were incubated with PBS instead of primary antibody.

Images were captured on a BX53 microscope (Olympus Corporation, Tokyo, Japan) using a QImaging MicroPublisher camera (Abingdon, Virginia, USA) and Q Capture software (VayTek Inc., Fairfield, IA, USA). To quantify $\mathrm{FOS}^{+}$neurons, a manual tagging feature of Image-Pro Plus was used. The dorsal horn portions were divided into three regions, including the laminae I-II, laminae III-IV, and laminae V-VI according to Molander's map of the adult rat spinal cord. ${ }^{25}$ We chose the segmental level of the tissue section using light field microscopy at $\times 100$ magnification and counted each stained $\mathrm{FOS}^{+}$neuron manually at $\times 200$ magnification.
The number of $\mathrm{FOS}^{+}$neurons for one rat was obtained as the mean of that in five L4-L5 sections selected randomly. In each group, the mean numbers of $\mathrm{FOS}^{+}$neurons in six rats were obtained similarly. Counting of $\mathrm{FOS}^{+}$neurons were performed by an investigator blinded to the drug treatment. We compared the numbers of $\mathrm{FOS}^{+}$cells in four groups at 2 hours, 8 hours, 2 days, and 7 days.

\section{Histopathology}

To examine the long-term changes of the nerves and tissues around, the surrounding ipsilateral sciatic nerves and tissues in Con, PELA, Rop, and Rop-PELA groups were removed at 7 days after drug administration, post fixed for 48 hours in the fixative solution used for perfusion (4\% buffered paraformaldehyde), and embedded in paraffin. The nerves and tissues were then cut into $4 \mu \mathrm{m}$ thick longitudinal sections and stained with hematoxylin and eosin. To observe systemic effects of the Rop-PELA, we assessed histopathology of the main organs (heart, liver, spleen, lung, kidney, and brain) of the rats in the Rop-PELA group at 7 days after administration.

\section{Statistics}

Statistical analysis was conducted using SPSS 16.0 (SPSS Inc., Chicago, IL, USA). Data were expressed as mean \pm standard deviation (SD). Drug And Statistics (DAS) 2.0 (Shanghai University of Traditional Chinese Medicine Clinical Drug Research Center, Shanghai, People's Republic of China) was used to compare the pharmacokinetics parameters between Rop and Rop-PELA in plasma. A two-way repeated measures analysis of variance with Bonferroni post hoc test was used to compare the differences in MWT and TWL over time between different groups. Immunohistochemical data among groups were analyzed using one-way analysis of variance followed by a Tukey's post hoc test. $P<0.05$ was considered statistically significant.

\section{Results \\ Drug absorption in vivo}

Our results showed that Rop-nanoparticles released 30\% Rop for the first 10 hours and continuously released it for over 7 days in in vitro study. ${ }^{19}$ In in vivo study, we found that mass concentration $(Y)$ and peak area $(X)$ had linearity from $25 \mathrm{ng} / \mathrm{mL}$ to $2,500 \mathrm{ng} / \mathrm{mL}$, and the linear equation was $Y=1.799 X+1.447$ (correlation coefficient $r=0.9998$ ). The parameters of pharmacokinetics between Rop and Rop-PELA groups were as follows: $C_{\text {max }}$ was $0.613 \mathrm{mg} / \mathrm{L}$ and $0.352 \mathrm{mg} / \mathrm{L}, T_{\max }$ was 0.5 hours and 1 hour, $K_{\mathrm{a}}$ was $2.755 /$ hour and 1.966/hour, $T_{1 / 2}$, abs was 0.252 hours and 0.353 hours, $T_{1 / 2}$, elimination was 0.919 hours 


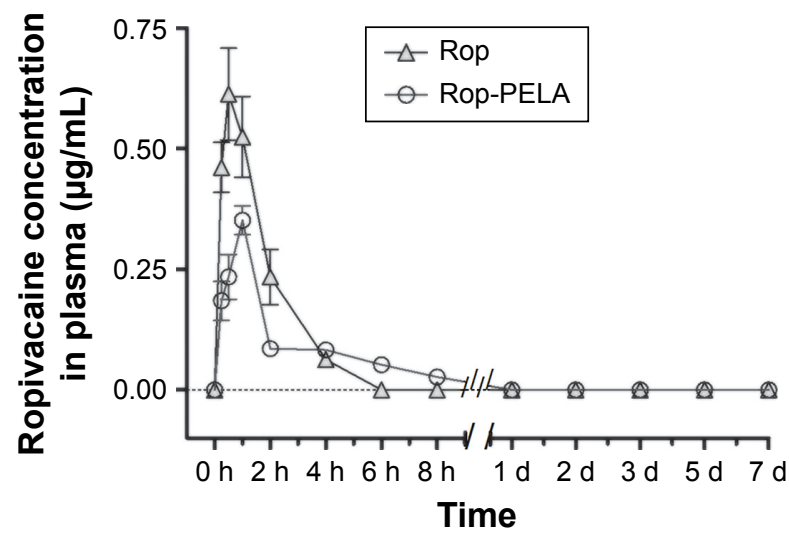

Figure I Plasma ropivacaine concentration in the ropivacaine group (Rop) and slow-released ropivacaine group (Rop-PELA).

Notes: Plasma ropivacaine concentrations in different groups were measured by high performance liquid chromatography. Data are shown as mean $\pm S D$ ( $n=6$, each).

Abbreviations: Rop, ropivacaine; PELA, polyethylene glycol-co-polylactic acid; h, hour; d, day; SD, standard deviation.

and 21.789 hours, $K_{\mathrm{e}}$ was 0.898 hours and 0.831 hours, and AUC was $1.165 \mathrm{mg} / \mathrm{L} \cdot \mathrm{h}(0-4)$ and $0.834 \mathrm{mg} / \mathrm{L} \cdot \mathrm{h}(0-8)$, respectively. The concentration of Rop in the Rop group was too low to be detected at 4 hours, whereas in the Rop-PELA group, Rop lasted for $>8$ hours (Figure 1).

\section{Mechanical withdrawal threshold}

The results of MWT in the Rop-PELA group were significantly higher than those in the other three groups (from 4 hours to 3 days; $P<0.05$ ). For the Rop group, significant difference was only produced at 2 hours after incision when compared with the Con group $(P<0.05)$. In addition, the MWT of the PELA group was similar to that of the Con group at all time points (Figure $2 \mathrm{~A}$ ).

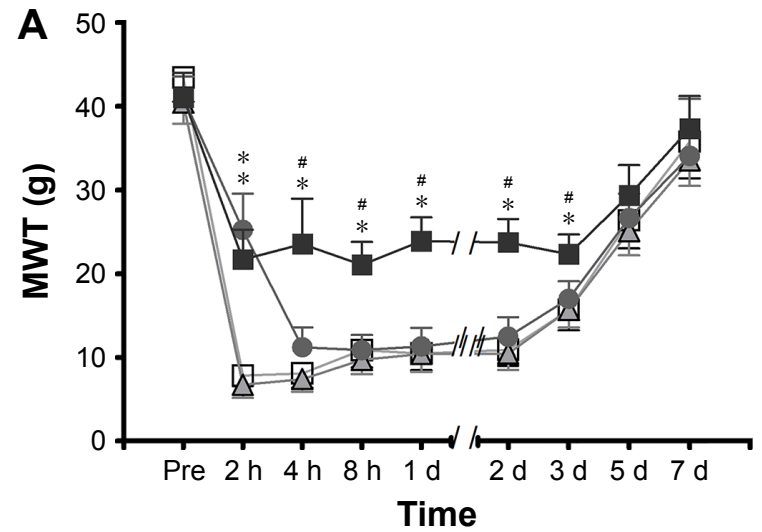

\section{Thermal withdrawal latency}

The results of TWL were similar to those of MWT (Figure 2B). The latency was decreased significantly in comparison with the baseline value (from 2 hours to 3 days). The Rop-PELA group showed longer antihyperalgesic effects than the Rop group ( 3 days vs 2 hours, $P<0.05$ ). There were no significant differences between the PELA group and Con group $(P>0.05)$.

\section{c-FOS immunoreactivity}

Compared with the Con group, numbers of $\mathrm{FOS}^{+}$neurons in the Rop-PELA group were lower in laminae I-II after 2 hours-2 days of incision $(P<0.05)$; as well as in laminae III-IV and V-VI after 8 hours and 2 days of incision $(P<0.05)$. However, the $\mathrm{FOS}^{+}$neurons in the Rop-PELA group was lower after 8 hours of incision in laminae I-II $(P<0.01)$ and V-VI compared with the Rop group $(P<0.05)$. Between the Rop group and Con group, there was no difference except after 2 hours of administration in laminae I-II $(P<0.01)$, laminae III-IV $(P<0.05)$, and laminae V-VI $(P<0.05$; Figure 3$)$.

\section{Behavioral, histopathological, and toxicity evaluation}

We did not observe any side effects in the PELA and RopPELA groups of rats, such as clinical seizures, respiratory distress, terminal apnea, as well as deaths. Slight foreign body (unabsorbed nanoparticles) and minority inflammation cells around PELA nanoparticles were observed till 7 days after administration (Figure 4). No obvious changes were investigated in the major organs with Rop-PELA administration for nerve block (Figure 5).

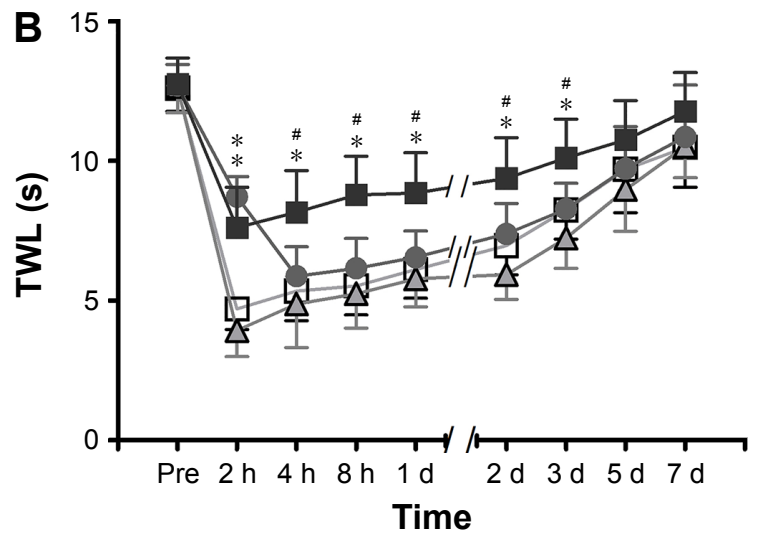

Figure 2 Results of behavioral testing of rats in control, PELA, ropivacaine and Rop-PELA groups.

Notes: (A) MWT (g); (B) TWL (seconds). Mechanical allodynia and thermal hyperalgesia were induced by hind paw incision. Data were expressed as mean \pm SD, $\mathrm{n}=5$ in each group. $* P<0.05$ compared with the control group. ${ }^{*} P<0.05$ compared with the ropivacaine group.

Abbreviations: Con, control; Rop, ropivacaine; PELA, polyethylene glycol-co-polylactic acid; MWT, mechanical withdrawal threshold; h, hour; d, day; TWL, thermal withdrawal latency; s, second. 

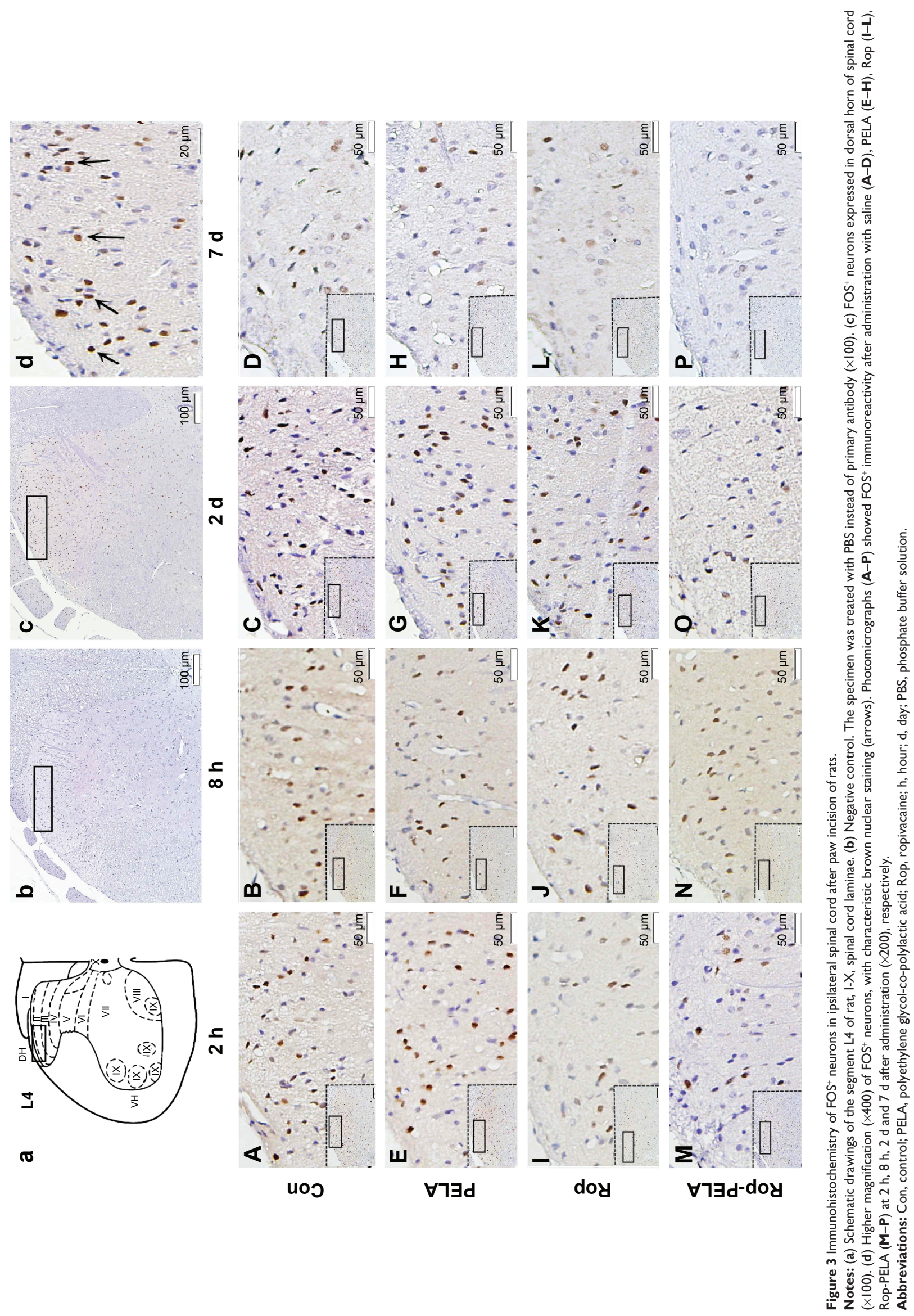

T
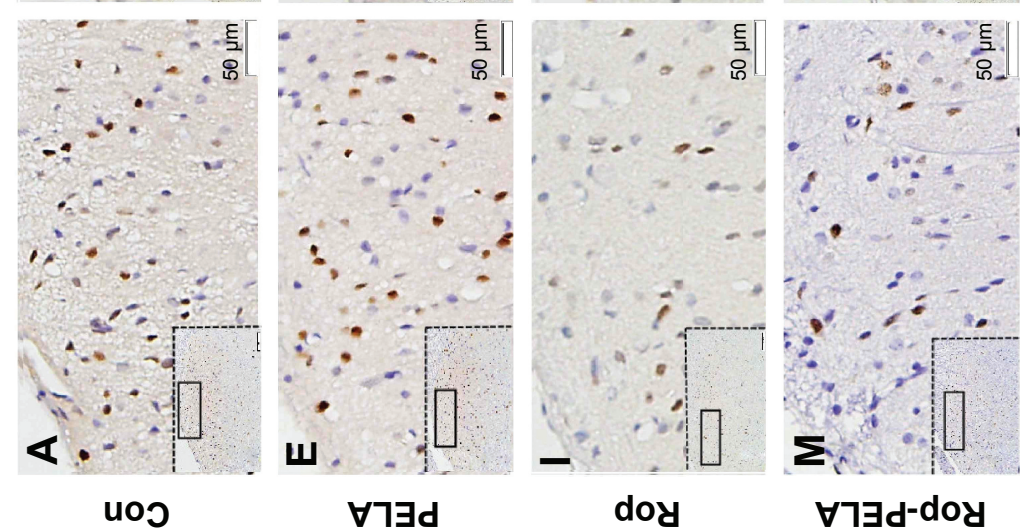

$\forall$ ㅋd-doy 

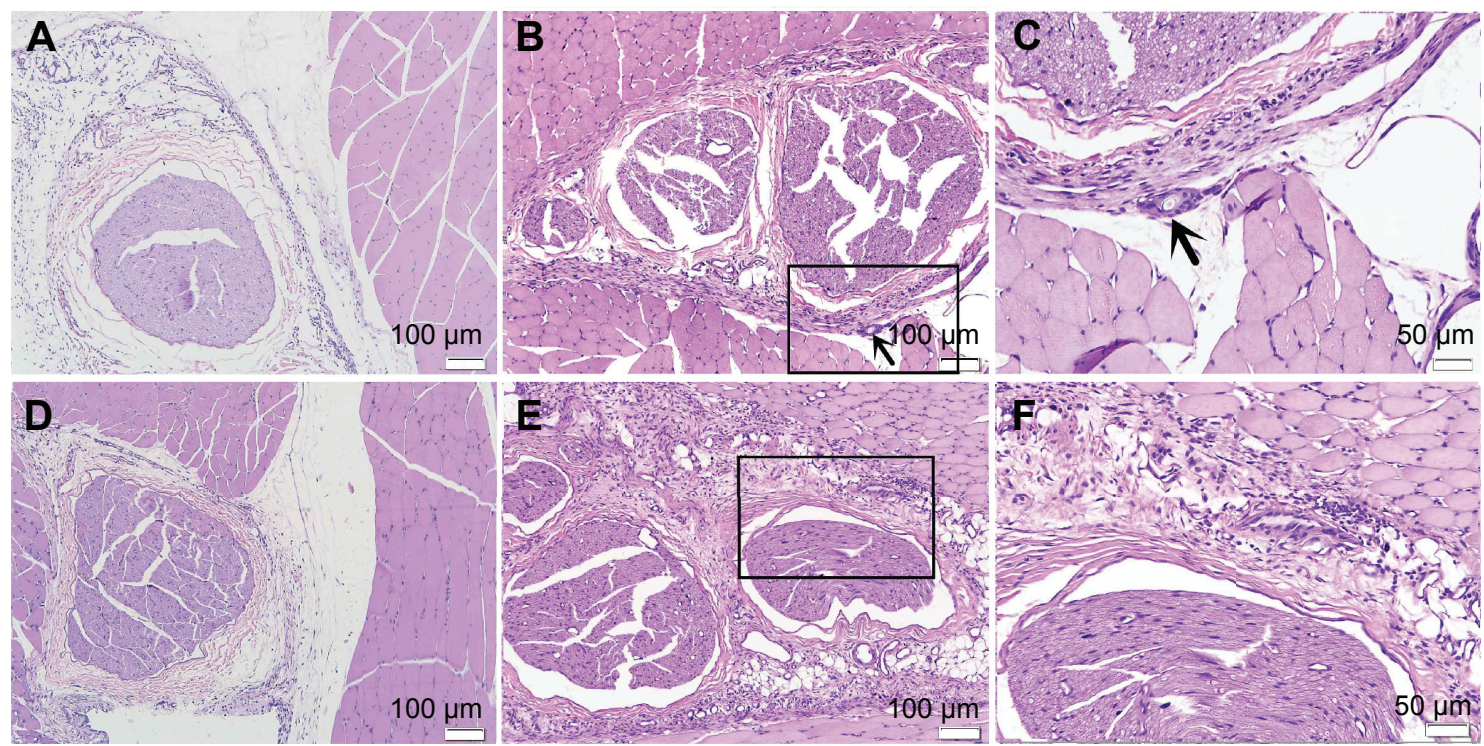

Figure 4 Sciatic nerves and surrounding tissues were stained with hematoxylin and eosin at $7 \mathrm{~d}$ after administration.

Notes: (A) Image of rats in control group, no inflammation cell was observed $(\times 100)$. (B) Image of rats treated with PELA, some inflammation cells were observed $(\times 100)$. (C) High-magnification images of (B), arrows to the inflammation cells and microparticles $(\times 200)$. (D) Image of rats in Rop group, slight inflammation was observed near the sciatic nerve $(\times \mid 00)$. (E) Image of rats in Rop-PELA group, granulation tissue and moderate degree inflammation were detected at the site of administration $(\times 100)$. $(\mathbf{F})$ High-magnification images of $(\mathbf{E})(\times 200)$. Sciatic nerves in all groups were almost normal. Abbreviations: Rop, ropivacaine; PELA, poly ethylene glycol-co-poly lactic acid; d, day.

\section{Discussion}

The present study demonstrates that plasma Rop released from PELA nanoparticles had rapid initial release of drug (burst effect) and could continuously release Rop in vivo for more than 8 hours. The behavioral testing results showed that Rop from nanoparticles could inhibit hyperalgesia caused by incision for over 3 days. Expression of c-FOS in the spinal cord was also suppressed significantly in the Rop-PELA group. No harm was observed in nerves and major organs at 7 days.

With the development of medical polymeric materials, sustained-release formulations of local anesthetics have been produced in various forms, ${ }^{20,27,28}$ including liposomes, ${ }^{29-31}$ poly(D,L-lactic acid; PLA) ${ }^{32}$ nanoparticles, and PLGA
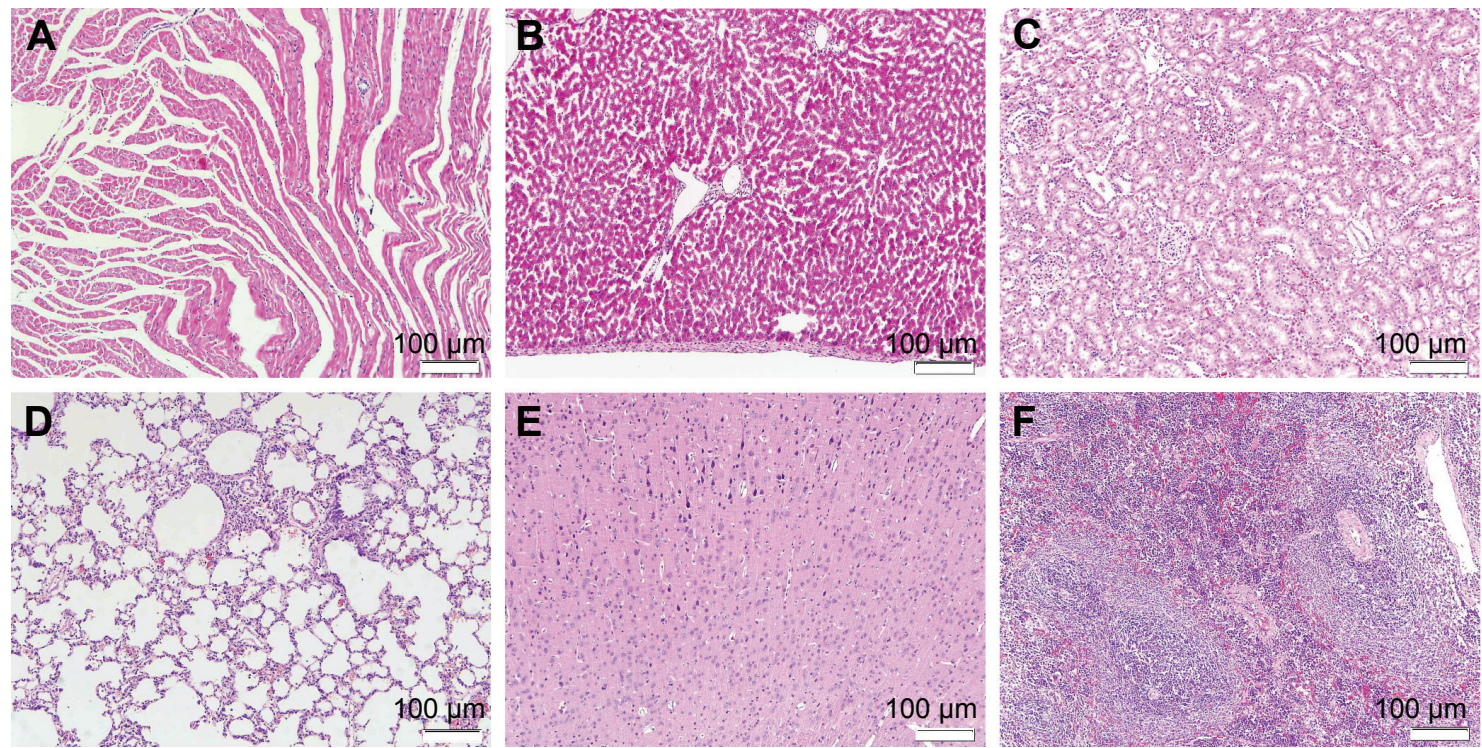

Figure 5 The major organs of rats in Rop-PELA group were sectioned and stained with hematoxylin and eosin $7 \mathrm{~d}$ after administration.

Notes: (A) Heart, (B) liver, (C) spleen, (D) lung, (E) kidney, (F) brain $(\times 100)$.

Abbreviations: Rop, ropivacaine; PELA, poly ethylene glycol-co-poly lactic acid; $d$, day. 
nanoparticles. ${ }^{13}$ PLA hydrolyzes into nontoxic hydroxylcarboxylic acid through ester bond cleavage in the aquatic environment and then is metabolized into water and carbon dioxide through a citric acid cycle. However, weaker hydrophilicity has prevented PLA and PLGA from achieving their full potential in drug delivery.

Theoretically, the slow-released drugs usually exhibit a "double peak" phenomenon at early stage after the drug administration. Previous data showed that sciatic nerve block with lidocaine caused plasma lidocaine concentration to increase sharply at the early stage (burst effect) and then decline to the baseline; however, slow-release lidocaine sheet maintained the plasma lidocaine concentration in a continuous low level, lacking the initial burst effect. ${ }^{13}$ Different from double peak phenomenon, we only observed a burst release peak both in Rop and Rop-PELA group, the second peak about Rop from Rop-PELA was not detected. These results are similar to the results of Tobe et al. ${ }^{13}$ The lower peak concentration in plasma and the longer duration could be due to the size of the particles and lower absorption surrounding the nerve. Indeed, we could not predict the dose of Rop realized at a given moment; therefore, the duration of action might not only be determined by the total dose of the Rop-PELA compound. Lack of the second peak plasma concentration was not due to the "noneffective" release but due to the lack of absorption of the Rop from the Rop-PELA compound, as well as metabolism of Rop at the local site. Large molecular weight decreased the absorption through the nearby blood vessels, and lack of diminished of nanoparticles due to its better histocompatibility.

As a widely used local anesthesia in clinics, the efficacy of Rop was broadly similar to bupivacaine. More importantly, it was preferred because of its reduced cerebral and cardiac toxic potential and its lower propensity for motor block. Thus, we imagined that peripheral approach for treatment of postoperative pain with long-acting Rop is exciting because it is inherently simple and targets pain at the site of origin before centrally mediated changes can occur in the spinal cord or the cerebral cortex.

Owing to the protrusion of the hydrophilic PEG segment to the surface of nanoparticles, a large burst release in the initial stage might exist as a previous study reported. ${ }^{19}$ In addition, particle size was $\sim 50 \mu \mathrm{m}$. These reasons could affect the release of the Rop in vivo. As has been mentioned by Polakovic et al, ${ }^{32}$ two main mechanisms determining the lidocaine release from the PLA matrix are diffusion and solid drug dissolution, but the drug release varies strongly with the parameters such as the molecular weight of nanoparticles, particle size, or drug content. Söderberg et a ${ }^{33}$ found that the high percentage formulations showed the longest duration of action yet reported for sciatic nerve block in rats. The researchers ${ }^{34}$ also indicated that increasing initial lidocaine content proportionately increased the duration of functional sciatic nerve block and single treatment with a slow-release lidocaine sheet $(30 \%, \mathrm{w} / \mathrm{w})$ inhibited hyperalgesia caused by paw incision for 1 week. ${ }^{13}$ In our study, Rop nanoparticles were used and its inhibition effect for hyperalgesia lasted for over 3 days. Despite the precise concentration of Rop released from nanoparticles into the space around the sciatic nerve being difficult to detect, the plasma concentration of Rop in the Rop-PELA group at 0.5 hours was approximately one-third of the peak concentration in the Rop group and only showed initial burst release, which is consistent with the result in vitro. Thus, we can predict that the duration would be longer when the drug content increased.

Allodynia and hyperalgesia were observed 5-7 days after incision, similar to that reported by Brennan et al. ${ }^{22}$ The group treated with Rop-PELA did show antihypersensitivity when compared with the control group for 3 days. There was no significant difference during the experimental period in the rats without Rop (Con and PELA). However, the duration of sciatic nerve block in behavioral testing was much longer than the absorption of Rop in plasma. We thought that the block length associated with the Rop-PELA could be due to the long-lasting local exposure of the nerve to Rop with the metabolism of the PLEA nanoparticles. Exposing the nerve to a higher concentration of local anesthetic was not the reason for its long-acting effect.

As one of the immediate early genes, c-FOS and its protein product FOS were expressed in cells rapidly and transiently after various forms of stimulation. It was reported that sensory inputs, especially nociceptive inputs, could increase expression of FOS. ${ }^{35,36}$ In the current study, inhibition of FOS expression was obviously in the Rop-PELA group for a long time. These results corresponded with those of others. ${ }^{13,37}$ In spite of dissociation between analgesics on pain behaviors and FOS expression in the spinal cord dorsal horn described by researchers, ${ }^{38,39}$ we found the FOS expression to correspond with the testing of pain behaviors. Therefore, the sustained release of Rop-PELA nanoparticles might be promising for the management of acute postoperative pain.

The pathogenesis of long-acting local analgesic-induced tissue toxicity is poorly understood. A previous study showed that PLA itself did not produce nerve inflammation or demyelination when used for the sustained release of bupivacaine. ${ }^{18}$ However, other researchers showed 
different views. One study reported that a slight foreign body reaction occurred around PLA nanoparticles and suggested that the reaction may cause local acidosis. ${ }^{40}$ Padera et al noted that myocardiotoxicity may be related to both the release kinetics of anesthetic (burst and duration of release) and the presence of the particles themselves. ${ }^{41}$ Ischemia is one of the possible causative mechanisms that may result from changes in peripheral blood flow. ${ }^{42}$ An immune-mediated mechanism may be possible as suggested by others. ${ }^{21,43}$ In the current study, we observed that the local inflammatory process associated with the Rop-PELA might be due to the stimuli by unabsorbed Rop nanoparticles. We did not observe the long-term side effects and safety of Rop-PELA on tissues and nerve toxicity at $\geq 2$ weeks -1 month.

We tested the behaviors of rats by electrical von Frey, which measured the paw withdrawal threshold at once and showed the results on a computer screen. Therefore, the need for finding the threshold by up-down method and the limitations of variability in the filament's strength were overcome in our study. In spite of this, the study had some limitations. First, the way of administration limits its clinical application. The nanoparticles were placed directly following surgical exposure. It might be suitable for surgical site, such as abdominal incisions. Second, we detected neural and organic changes by routine histologic examination using a light microscope, but the toxic effects of nanoparticles on muscle cells, neurons, and Schwann cells should be detected by specialized imaging with either high-powered light microscopy or electron microscopy. Thus, more research should be done in the future. Drug Rop released from the nanoparticles should be controlled by designing different proportion of PEG and PLA, to meet the demands for postoperative pain management. Moreover, the dosage form of Rop nanoparticles for injection should be developed for the requirement of clinical application.

\section{Conclusion}

The Rop-PELA nanoparticles produced an analgesic effect for nerve blocking for over 3 days after single administration, and the analgesic mechanism might be mediated by the regulation of spinal c-FOS expression. However, the long-term safety of Rop-PELA nanoparticles on tissues or nerves needs to be further investigated.

\section{Acknowledgments}

This research was supported by the National Natural Science Foundation of China (No 81171864), the Fundamental Research Funds of Shandong University (No 2014YQ014), the Jinan City High School Innovative Plan (No 201102071), and the Jinan City Study Abroad Personnel Poineering Plan (No 20110408). The authors would like to thank Professor Qingzeng Zhu from the School of Chemistry, Shandong University, for synthesizing ropivacaine nanoparticles.

\section{Author contributions}

All authors contributed toward data analysis, drafting and critically revising the paper and agree to be accountable for all aspects of the work.

\section{Disclosure}

The authors report no conflicts of interest in this work.

\section{References}

1. White $\mathrm{PF}, \mathrm{Kehlet} \mathrm{H}$. Improving postoperative pain management: what are the unresolved issues? Anesthesiology. 2010;112(1):220-225.

2. Apfelbaum JL, Chen C, Mehta SS, Gan TJ. Postoperative pain experience: results from a national survey suggest postoperative pain continues to be undermanaged. Anesth Analg. 2003;97(2):534-540.

3. Carli F, Mayo N, Klubien K, Schricker T, Trudel J, Belliveau P. Epidural analgesia enhances functional exercise capacity and healthrelated quality of life after colonic surgery: results of a randomized trial. Anesthesiology. 2002;97(3):540-549.

4. Liu SS, Wu CL. Effect of postoperative analgesia on major postoperative complications: a systematic update of the evidence. Anesth Analg. 2007;104(3):689-702.

5. Macrae WA. Chronic post-surgical pain: 10 years on. Br J Anaesth. 2008; 101(1):77-86.

6. Kehlet H, Jensen TS, Woolf CJ. Persistent postsurgical pain: risk factors and prevention. Lancet. 2006;367(9522):1618-1625.

7. Borgeat A, Ekatodramis G, Schenker CA. Postoperative nausea and vomiting in regional anesthesia: a review. Anesthesiology. 2003;98(2): 530-547.

8. Govender T, Riley T, Ehtezazi T, et al. Defining the drug incorporation properties of PLA-PEG nanoparticles. Int J Pharm. 2000;199(1): 95-110.

9. Li Y, Pei Y, Zhang X, et al. PEGylated PLGA nanoparticles as protein carriers: synthesis, preparation and biodistribution in rats. J Control Release. 2001;71(2):203-211.

10. Katragadda U, Teng Q, Rayaprolu BM, et al. Multi-drug delivery to tumor cells via micellar nanocarriers. Int J Pharm. 2011;419(1-2):281-286.

11. Li X, Zhang Y, Yan R, et al. Influence of process parameters on the protein stability encapsulated in poly-DL-lactide-poly ethylene glycol microspheres. J Control Release. 2000;68(1):41-52.

12. Mosqueira VC, Legrand P, Gulik A, et al. Relationship between complement activation, cellular uptake and surface physicochemical aspects of novel PEG-modified nanocapsules. Biomaterials. 2001;22:2967-2979.

13. Tobe M, Obata H, Suto T, et al. Long-term effect of sciatic nerve block with slow-release lidocaine in a rat model of postoperative pain. Anesthesiology. 2010;112(6):1473-1481.

14. Fredrickson MJ, Krishnan S, Chen CY. Postoperative analgesia for shoulder surgery: a critical appraisal and review of current techniques. Anaesthesia. 2010;65(6):608-624.

15. Ilfeld BM, Thannikary LJ, Morey TE, et al. Popliteal sciatic perineural local anesthetic infusion: a comparison of three dosing regimens for postoperative analgesia. Anesthesiology. 2004;101(4):970-977.

16. Mulroy MF, Larkin KL, Batra MS, et al. Femoral nerve block with $0.25 \%$ or $0.5 \%$ bupivacaine improves postoperative analgesia following outpatient arthroscopic anterior cruciate ligament repair. Reg Anesth Pain Med. 2001;26(1):24-29. 
17. Sokolsky-Papkov M, Golovanevski L, Domb AJ, et al. Prolonged local anesthetic action through slow release from poly (lactic acid co castor oil). Pharm Res. 2009;26(1):32-39.

18. Sokolsky-Papkov M, Golovanevski L, Domb AJ, et al. Poly (DL: lactic acid-castor oil) 3:7-bupivacaine formulation: reducing burst effect prolongs efficacy in vivo. J Pharm Sci. 2010;99(6):2732-2738.

19. Yao B, Zhu Q, Liu H, Qiao L, Hao J, Qi F. Conformation and aggregation behavior of poly (ethylene glycol)-b-poly (lactic acid) amphiphilic copolymer chains in dilute/semidilute THF solutions. J Appl Polym Sci. 2012;125(supp1 2):E223-E230.

20. Yin QQ, Wu L, Gou ML, Qian ZY, Zhang WS, Liu J. Long-lasting infiltration anaesthesia by lidocaine-loaded biodegradable nanoparticles in hydrogel in rats. Acta Anaesthesiol Scand. 2009;53(9):1207-1213.

21. Brummett CM, Norat MA, Palmisano JM, Lydic R. Perineural administration of dexmedetomidine in combination with bupivacaine enhances sensory and motor blockade in sciatic nerve block without inducing neurotoxicity in the rat. Anesthesiology. 2008;109(3):502-511.

22. Brennan TJ, Vandermeulen EP, Gebhart GF. Characterization of a rat model of incisional pain. Pain. 1996;64(3):493-501.

23. Kawata T, Homma M, Kakiuchi Y, et al. Liquid chromatographic determination of plasma ropivacaine for assessing pharmacokinetics of the viscous preparation. Biol Pharm Bull. 2005;28(12):2271-2273.

24. Wang C, Ning LP, Wang YH, et al. Nuclear factor-kappa B mediates TRPV4-NO pathway involved in thermal hyperalgesia following chronic compression of the dorsal root ganglion in rats. Behav Brain Res. 2011;221(1):19-24.

25. Hargreaves K, Dubner R, Brown F, Flores C, Joris J. A new and sensitive method for measuring thermal nociception in cutaneous hyperalgesia. Pain. 1988;32(1):77-88.

26. Molander C, Xu Q, Grant G. The cytoarchitectonic organization of the spinal cord in the rat. I. The lower thoracic and lumbosacral cord. J Comp Neurol. 1984;230(1):133-141.

27. Ratajczak-Enselme M, Estebe JP, Dollo G, et al. Epidural, intrathecal and plasma pharmacokinetic study of epidural ropivacaine in PLGAmicrospheres in sheep model. Eur J Pharm Biopharm. 2009;72(1): 54-61.

28. Chen PC, Park YJ, Chang LC, et al. Injectable microparticle-gel system for prolonged and localized lidocaine release. I. In vitro characterization. J Biomed Mater Res A. 2004;70(3):412-419.

29. Grant SA. The Holy Grail: long-acting local anaesthetics and liposomes. Best Pract Res Clin Anaesthesiol. 2002;16(2):345-352.

30. Grant GJ, Barenholz Y, Bolotin EM, et al. A novel liposomal bupivacaine formulation to produce ultralong-acting analgesia. Anesthesiology. 2004; 101(1):133-137.
31. Boogaerts JG, Lafont ND, Luo H, et al. Plasma concentrations of bupivacaine after brachial plexus administration of liposome-associated and plain solutions to rabbits. Can J Anaesth. 1993;40(12):1201-1204.

32. Polakovic M, Gorner T, Gref R, et al. Lidocaine loaded biodegradable nanospheres. II. Modelling of drug release. J Control Release. 1999; 60(2-3):169-177.

33. Söderberg L, Dyhre H, Roth B, Björkman S. Ultralong peripheral nerve block by lidocaine: prilocaine 1:1 mixture in a lipid depot formulation: comparison of in vitro, in vivo, and effect kinetics. Anesthesiology. 2006;104(1):110-121.

34. Gerner P, Wang CF, Lee BS, et al. The relationship between functional sciatic nerve block duration and the rate of release of lidocaine from a controlled-release matrix. Anesth Analg. 2010;111(1):221-229.

35. Hunt SP, Pini A, Evan G. Induction of c-fos-like protein in spinal cord neurons following sensory stimulation. Nature. 1987;328(6131): 632-634.

36. Presley RW, Menetrey D, Levine JD, et al. Systemic morphine suppresses noxious stimulus-evoked Fos protein-like immunoreactivity in the rat spinal cord. J Neurosci. 1990;10(1):323-335.

37. Sun X, Yokoyama M, Mizobuchi S, et al. The effects of pretreatment with lidocaine or bupivacaine on the spatial and temporal expression of c-Fos protein in the spinal cord caused by plantar incision in the rat. Anesth Analg. 2004;98(4):1093-1098.

38. Yashpal K, Mason P, McKenna JE, et al. Comparison of the effects of treatment with intrathecal lidocaine given before and after formalin on both nociception and Fos expression in the spinal cord dorsal horn. Anesthesiology. 1998;88(1):157-164.

39. Gilron I, Quirion R, Coderre TJ. Pre-versus postformalin effects of ketamine or large-dose alfentanil in the rat: discordance between pain behavior and spinal Fos-like immunoreactivity. Anesth Analg. 1999;89(1):128-135.

40. Drager C, Benziger D, Gao F, et al. Prolonged intercostal nerve blockade in sheep using controlled-release of bupivacaine and dexamethasone from polymer microspheres. Anesthesiology. 1998;89(4):969-979.

41. Padera R, Bellas E, Tse JY, et al. Local myotoxicity from sustained release of bupivacaine from microparticles. Anesthesiology. 2008; 108(5):921-928.

42. Hashimoto K, Hampl KF, Nakamura Y, et al. Epinephrine increases the neurotoxic potential of intrathecally administered lidocaine in the rat. Anesthesiology. 2001;94(5):876-881.

43. Zink W, Seif C, Bohl JR, et al. The acute myotoxic effects of bupivacaine and ropivacaine after continuous peripheral nerve blockades. Anesth Analg. 2003;97(4):1173-1179.
International Journal of Nanomedicine

\section{Publish your work in this journal}

The International Journal of Nanomedicine is an international, peerreviewed journal focusing on the application of nanotechnology in diagnostics, therapeutics, and drug delivery systems throughout the biomedical field. This journal is indexed on PubMed Central, MedLine, CAS, SciSearch ${ }^{\circledR}$, Current Contents ${ }^{\circledR} /$ Clinical Medicine,
Dovepress

Journal Citation Reports/Science Edition, EMBase, Scopus and the Elsevier Bibliographic databases. The manuscript management system is completely online and includes a very quick and fair peer-review system, which is all easy to use. Visit http://www.dovepress.com/ testimonials.php to read real quotes from published authors. 